\title{
Proportion of High-Risk/Very High-Risk Patients in Europe with Low-Density Lipoprotein Cholesterol at Target According to European Guidelines: A Systematic Review
}

Eric Bruckert · Klaus Georg Parhofer · Jose Ramon Gonzalez-Juanatey •

Børge Nordestgaard · Marcello Arca · Periklis Giovas · Kausik Ray

Received: December 5, 2019 / Published online: March 21, 2020

(C) The Author(s) 2020

\section{ABSTRACT}

Objective: Assess achievement of low-density lipoprotein cholesterol (LDL-C) targets in European Society of Cardiology (ESC)/European Atherosclerosis Society (EAS) guidelines.

Enhanced Digital Features To view enhanced digital features for this article go to https://doi.org/10.6084/ m9.figshare.11908161.

Electronic supplementary material The online version of this article (https://doi.org/10.1007/s12325020-01285-2) contains supplementary material, which is available to authorized users.

E. Bruckert $(\square)$

Endocrinologie Métabolisme et Prévention

Cardiovasculaire, Institut E3M et IHU

Cardiométabolique (ICAN), Hôpital Pitié Salpêtrière,

Paris, France

e-mail: eric.bruckert@aphp.fr

K. G. Parhofer

Medizinische Klinik IV-Grosshadern, Klinikum der

Universität München, Marchioninistr. 15, 81377

Munich, Germany

J. R. Gonzalez-Juanatey

Cardiology and Intensive Cardiac Care Department, CIBERCV, University Hospital, Santiago de

Compostela, Spain

B. Nordestgaard

Department of Clinical Biochemistry, Faculty of Health and Medical Sciences, Herlev and Gentofte Hospital, Copenhagen University Hospital,

University of Copenhagen, Copenhagen, Denmark
Design: Systematic literature review.

Data Sources: Medline, EMBASE, Cumulated Index to Nursing and Allied Health Literature. Eligibility Criteria: Observational studies reporting LDL-C levels/target attainment, measured between 1 August 2006 to 31 August 2017, in European adults with established cardiovascular disease (CVD), diabetes with target organ damage, familial hypercholesterolaemia (FH) or 10-year risk of fatal CVD $\geq 5 \%$ (assessed by Systematic Coronary Risk Evaluation [SCORE]).

Data Extraction and Synthesis: Two reviewers independently extracted relevant studies and

\author{
M. Arca \\ Department of Internal Medicine and Medical \\ Specialties, UOS Atherosclerosis Center, La Sapienza \\ University of Rome, Rome, Italy \\ P. Giovas \\ Amgen Europe GmbH, Rotkreuz, Switzerland \\ K. Ray \\ Department of Public Health and Primary Care, \\ Imperial Centre for Cardiovascular Disease \\ Prevention, Imperial College London, London, UK
}


assessed study quality using the Risk of Bias for Non-Randomised Studies-Interventions (ROBINS-I) tool. Primary outcome was the proportion of patients achieving LDL-C targets in the 2011/2016 ESC/EAS guidelines. Where available, patient characteristics were presented as means weighted by sample size. The proportions of patients achieving LDL-C targets in the 5 years before and after publication of the 2011 guidelines were compared using a chi-square test.

Results: Across 81 eligible studies (303,534 patients), achievement of LDL-C $<1.8 \mathrm{mmol} / \mathrm{L}$ was poor among patients with established CVD (16\%; range $9-56 \%)$ and at very high risk of CVD (SCORE $\geq 10 \%$ [18\%; 14-25\%]). In individuals with $\mathrm{FH}$, SCORE $5-10 \%$, or diabetes and target organ damage, LDL-C $<2.5 \mathrm{mmol} / \mathrm{L}$ was achieved by $15 \%(9-22 \%), 46 \%(21-55 \%)$ and $13 \%(6-34 \%)$, respectively. Comparing the 5 years before/after publication of the 2011 guidelines, target achievement increased significantly over time but remained suboptimal (LDL-C $<1.8,22 \%$ versus $15 \%$; LDL-C $<2.5$, $68 \%$ versus $61 \%$; both $p<0.001$; established CVD group only).

Conclusions: These data show suboptimal LDL$\mathrm{C}$ control among European patients at high risk of CVD. Those at greatest overall risk (clinically established CVD or at least a 10\% 10-year risk of fatal CVD) had the lowest achievement of 2011/2016 EAS/ESC LDL-C targets. With lower LDL-C targets advocated in 2019 ESC/EAS guidelines, this unmet need will increase.

Protocol Registration: PROSPERO registration number; CRD77844

Keywords: Cardiovascular disease; Guidelines; High-risk; LDL-C targets; Low-density lipoprotein cholesterol; Systematic review

\section{Key Summary Points}

High levels of low-density lipoprotein cholesterol (LDL-C) are a known risk factor for cardiovascular (CV) events and risk-based LDL-C targets are central to dyslipidaemia treatment guidelines.
This systematic review describes data from 81 observational studies reporting LDL-C levels measured between 2006 and 2017, and attainment of LDL-C targets set out in 2016 EAS/ESC dyslipidaemia guidelines, among European patients at high or very high risk of cardiovascular disease (CVD).

Few patients at high or very high risk of $\mathrm{CV}$ events achieved recommended LDL-C target levels. Those at the greatest overall risk, including those with a greater than $10 \%$ 10-year risk of fatal CVD (as assessed by SCORE) or clinically established CVD, had the lowest achievement of LDL-C targets.

These findings indicate a significant unmet need in LDL-C control among patients at high/very high risk of $\mathrm{CV}$ events and offer an opportunity to attenuate $\mathrm{CV}$ events on a population level.

Recent 2019 ESC/EAS dyslipidaemia guidelines recommend LDL-C levels be lowered as much as possible to prevent CVD, and the unmet need in high/very high-risk patients will be even greater when assessed against these new guideline targets.

\section{INTRODUCTION}

Cardiovascular disease (CVD) is one of the leading causes of morbidity and mortality across Europe, with high levels of low-density lipoprotein cholesterol (LDL-C) widely recognized as a risk factor for CV events [1]. Trials of statin therapy have shown that the benefits of reducing the risk of $\mathrm{CV}$ events are proportional to the magnitude of LDL-C lowering [2, 3]. Moreover, LDL-C targets are central to the guidance from the European Society of Cardiology (ESC)/European Atherosclerosis Society (EAS) on the treatment of dyslipidaemias, which recommends overall risk assessment and sets out therapeutic targets for LDL-C [1, 4-6]. 
While statin trials have shown that reducing LDL-C levels attenuates CVD events in at-risk individuals, driven largely by a reduction in rates of myocardial infarction (MI), the application of current guidelines on a population level in Europe is poorly understood. In the L-TAP 2 study, conducted across Asia, Europe, Latin America and North America, 9955 patients with dyslipidaemia on stable lipidlowering therapy were enrolled [7]. Achievement of regional LDL-C targets was $86 \%$ in lowrisk, $74 \%$ in moderate-risk and $67 \%$ in high-risk patients, suggesting that the greatest unmet need for LDL lowering was in patients at the highest overall risk for CVD events.

The main objective of this study was to describe the proportion of individuals in Europe, in four selected patient cohorts at high/ very high CVD risk, reaching the LDL-C targets recommended in the 2011 and 2016 ESC/EAS guidelines (Table 1). These groups are (1) established CVD, (2) diabetes mellitus (DM)

Table 1 European Society of Cardiology (ESC)/European Atherosclerosis Society (EAS) LDL-C targets for high/very high-risk groups

\begin{tabular}{lc}
\hline Clinical risk categories & Target LDL-C levels \\
\hline Established CVD & LDL-C $<1.8 \mathrm{mmol} / \mathrm{L}$ or \\
& $50 \%$ reduction in LDL-C \\
DM with target organ & LDL-C $<1.8 \mathrm{mmol} / \mathrm{L}$ or \\
damage & $50 \%$ reduction in LDL-C \\
FH & LDL-C $<2.5 \mathrm{mmol} / \mathrm{L}$ or \\
& maximal reduction in \\
& LDL-C with any possible \\
& drug combination plus \\
& LDL apheresis \\
SCORE $\geq 10 \%$ considered & Very high risk, LDL- \\
very high risk, SCORE & C $<1.8$ mmol/L or $50 \%$ \\
$5-10 \%$ considered high & reduction in LDL-C; high \\
risk & risk, LDL- \\
& C $<2.5$ mmol/L
\end{tabular}

$C V D$ cardiovascular disease, $L D L-C$ low-density lipoprotein cholesterol, $D M$ diabetes mellitus, $F H$ familial hypercholesterolaemia, SCORE Systematic COronary Risk Evaluation calculates 10-year risk of fatal cardiovascular disease as a percentage with target organ damage, (3) familial hypercholesterolaemia (FH) and (4) individuals with at least a 5\% 10-year risk of fatal CVD as determined by the Systematic Coronary Risk Evaluation (SCORE) tool [8]. A secondary objective was to evaluate whether the publication of the guidelines in 2011 resulted in an improvement of LDL-C control in these risk groups.

\section{METHODS}

This systematic review was conducted according to the protocol registered with PROSPERO (Registration number CRD77844) and in accordance with PRISMA and MOOSE guidelines $[9,10]$.

\section{Eligibility}

The following studies were included: (1) observational studies; (2) reporting LDL-C levels or LDL-C target attainment in European patients measured between 1 August 2006 and $31 \mathrm{Au}$ gust 2016; (3) with at least 200 individuals in one of the high/very high-risk cohorts defined in Table 1 (and as per 2011 ESC/EAS guidelines [5]; the LDL-C targets recommended in the 2016 update of these guidelines did not change substantially $[1,4]) ;(4)$ reporting exclusively on individuals aged 18 years or more. All studies not meeting these pre-specified inclusion criteria were excluded. Where studies reported on mixed cohorts or patients outside Europe, studies were included if at least $80 \%$ of the overall study population met the cohort definition or were from within Europe, respectively.

\section{Search Strategy}

Two reviewers independently searched Medline, EMBASE and Cumulated Index to Nursing and Allied Health Literature (CINAHL) for Medical Subject Heading $(\mathrm{MeSH})$ and keywords related to diabetes mellitus (DM), established CVD, familial hypercholesterolaemia (FH), SCORE and LDL-C (Supplementary Appendix 1). These searches were combined with a search strategy to identify studies reporting observational data 
in humans and supplemented by examining reference lists of included studies, reviews and meta-analyses [11, 12]. No language restrictions were applied.

\section{Selection of Studies and Data Extraction}

Two reviewers independently screened titles and abstracts to identify articles suitable for full text review, independently reviewed the full text articles, and extracted data on the following variables (where available, and using a predefined data extraction form [Supplementary Appendix 2]): study details (design, location, number of patients in EAS/ESC high-risk group reported); patient characteristics (prevalent DM, hypertension, active smoking, BMI, statin therapy, use of any lipid-lowering therapy); and outcomes (mean and range of LDL-C level, achievement of LDL-C targets $<2.5$ or $<1.8 \mathrm{mmol} / \mathrm{L}$, myocardial infarction, stroke, major adverse cardiovascular events [MACE], all-cause mortality). Any conflicts during the PRISMA process were to be resolved by the lead author (EB). Where studies reported duplicate data, the most recent report from the same cohort was included to reflect contemporary practice and increase power.

\section{Assessment of Risk of Bias}

The two reviewers independently assessed the quality of the included studies using the Risk of Bias for Non-Randomised Studies-Interventions (ROBINS-I) tool [13]. This tool assesses bias across seven domains: (1) bias due to confounding, (2) bias in selection of participants, (3) bias in classification of interventions, (4) bias due to deviations from intended interventions, (5) bias due to missing data, (6) bias in measurement of outcomes and (7) bias in selection of the reported result. Overall risk of bias was defined as low, moderate, serious or critical. Sensitivity analyses excluding studies with serious or critical risk of bias for the primary and secondary endpoint were planned.

\section{Statistical Analyses}

The primary outcome measure was the proportion of patients achieving LDL-C targets, as defined in the ESC/EAS 2011 (and 2016) guidelines. The proportion of patients achieving LDL$\mathrm{C}$ targets in the 5-year periods before and after publication of the $2011 \mathrm{ESC} / \mathrm{EAS}$ guideline, i.e. 1 August 2006-31 July 2011 and 1 August 2011-31 August 2016, respectively, were compared using a chi-square test. Where available, summary characteristics of participants, including LDL-C levels, are presented as mean values weighted by study size. Where LDL-C levels were reported at multiple time points during the 5-year periods before or after the publication of the 2011 ESC/EAS guideline, the most recent values were extracted to reflect contemporary practice. Secondary outcome measures were myocardial infarction, stroke and all-cause mortality. Where not directly reported for these secondary outcomes, event rates per 1000 person-years were calculated by dividing the absolute number of events by the total person-years of follow-up.

\section{Compliance with Ethics Guidelines}

There was no patient or public involvement in this research project. This article is based on previously conducted studies and does not contain any studies with human participants or animals performed by any of the authors.

\section{RESULTS}

\section{Search Strategy}

Of 17,683 potentially relevant publications, 915 studies were identified for full text review. After 47 duplicates were removed, a further 787 studies did not meet the eligibility criteria and were excluded; a total of 81 studies were included in the final analyses (Fig. 1). 


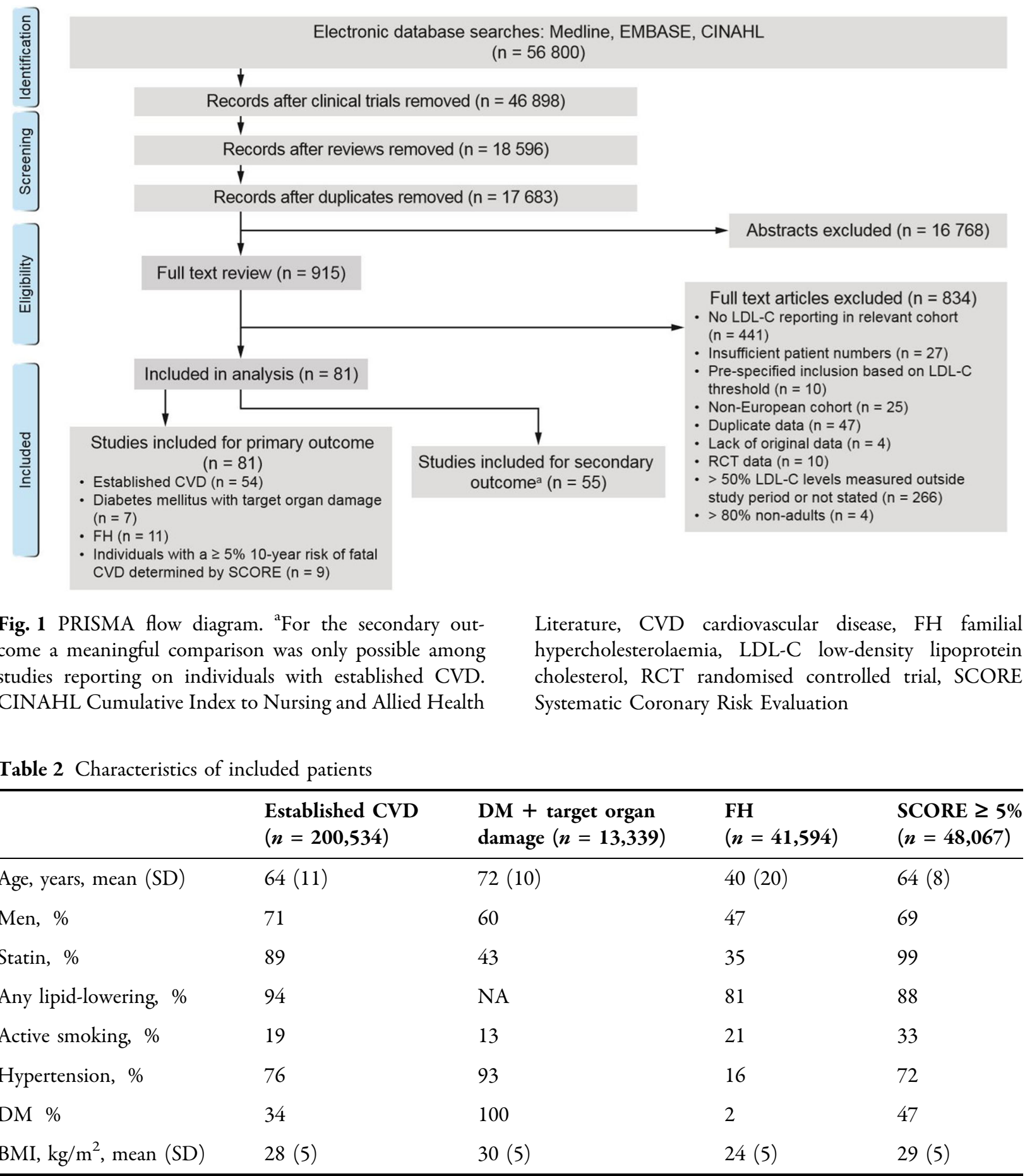

Mean values are weighted by study size

CVD cardiovascular disease, DM diabetes mellitus, FH familial hypercholesterolaemia, SCORE Systematic COronary Risk Evaluation, $N A$ data not available, $B M I$ body mass index, $S D$ standard deviation 


\section{Overview of Included Studies}

Included studies reported on a total of 303,534 patients across the four high/very high-risk groups: $n=200,534$ patients with established CVD, $n=13,339 \mathrm{DM}$ with target organ damage, $n=41,594 \mathrm{FH}$ and $n=48,067 \mathrm{SCORE} \geq 5 \%$. All studies reported LDL-C levels and/or achievement of LDL-C targets while patients were receiving lipid modification therapy. The majority of included studies were based on registry data or cohort studies (51 studies, 63\%); 30 studies (37\%) were cross-sectional in design (Supplementary Appendices 2 and 3). Characteristics of included patients are presented in Table 2. The definition of FH used in included studies is presented in Supplementary Appendix 4. Compared with other high/very high-risk groups, those with $\mathrm{FH}$ were younger, less likely to be hypertensive or diabetic, and had a lower body mass index. Statin use was frequent among patients with established CVD $(89 \%)$ and SCORE $\geq 5 \%$ (99\%); whereas only a minority of patients with FH (35\%) or DM with target organ damage (43\%) were receiving a statin.

\section{Risk of Bias}

The overall risk of bias, measured using the Robins-I tool, was moderate for all included studies; hence, planned sensitivity analyses excluding studies with serious or critical risk of bias were not performed (Supplementary
Appendix 5). No study selected participants on the basis of LDL-C measurements after the study start. Our analyses did not assess the effect size of any intervention on the primary or secondary outcome measures; therefore, no study was considered to have deviated from an intended intervention. Baseline characteristics were reported in the overall population of all studies; however, in six studies our inclusion criteria only applied to one or more subgroups where these characteristics were not reported. No bias in the measurement of outcome was observed in any of the included studies.

\section{Statistical Analysis}

Pooled estimates for mean LDL-C levels and the proportion of patients achieving EAS/ESC target LDL-C levels are presented in Table 3 . Across the four high/very high-risk groups, mean LDL-C levels were lowest among patients with established CVD (weighted mean $2.8 \mathrm{mmol} / \mathrm{L}$, SD 0.9 ) and highest among those with $\mathrm{FH}$ (weighted mean $4.9 \mathrm{mmol} / \mathrm{L}$, SD 1.9).

The ESC/EAS recommended target LDL-C level of $<1.8 \mathrm{mmol} / \mathrm{L}$ for patients with established CVD was achieved in only 16\% (range $9-56 \%)$ of patients included in this cohort. No study including patients with DM and target organ damage reported on achievement of the EAS/ESC recommended LDL-C target level of $<1.8 \mathrm{mmol} / \mathrm{L}$; however, achievement of the less stringent LDL-C target of $<2.5$ was similarly low at $13 \%$ (range 6-36\%). Among the

Table 3 Mean LDL-C levels and target achievement for high/very high-risk groups

\begin{tabular}{lllll}
\hline & $\begin{array}{l}\text { Established CVD } \\
(\boldsymbol{n}=\mathbf{2 0 0 , 5 3 4})\end{array}$ & $\begin{array}{l}\text { DM + target organ } \\
\text { damage }(\boldsymbol{n}=\mathbf{1 3 , 3 3 9 )})\end{array}$ & $\begin{array}{l}\text { FH } \\
(\boldsymbol{n}=\mathbf{4 1 , 5 9 4})\end{array}$ & $\begin{array}{l}\text { SCORE } \geq \text { 5\% } \\
(\boldsymbol{n}=\mathbf{4 8 , 0 6 7})\end{array}$ \\
\hline $\begin{array}{l}\text { Mean LDL-C, mmol/L (SD) } \\
\text { LDL-C target achievement }\end{array}$ & $2.8(0.9)$ & $3.0(0.9)$ & $4.9(1.9)$ & $2.9(1.0)$ \\
$<1.8 \mathrm{mmol} / \mathrm{L}, \%$ (range) & $16(9-56)$ & NA & - & $16(14-25)$ \\
$<2.5 \mathrm{mmol} / \mathrm{L}, \%$ (range) & $66(24-81)$ & $13(6-36)$ & $15(9-22)$ & $29(5-63)$ \\
\hline
\end{tabular}

Mean values are weighted by study size

$L D L-C$ low density lipoprotein cholesterol, $C V D$ cardiovascular disease, $D M$ diabetes mellitus, $F H$ familial hypercholesterolaemia, SCORE Systematic COronary Risk Evaluation, $N A$ not available (no study reported these data), - not applicable to this cohort 


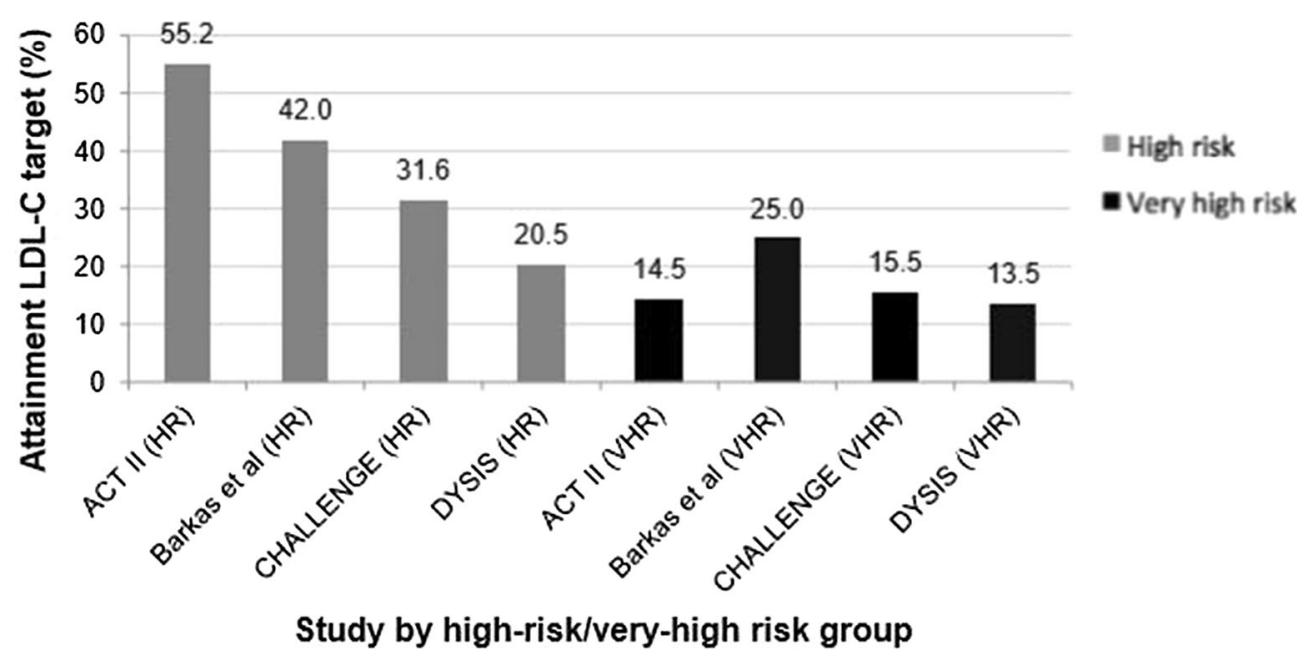

Fig. 2 LDL-C goal achievement among patients with SCORE $5-10 \%$ (high risk) and SCORE $\geq 10 \%$ (very high risk). Studies included in this figure were those

mixed primary and secondary prevention cohort of patients with $\mathrm{FH}$, achievement of the recommended LDL-C target of $<2.5 \mathrm{mmol} / \mathrm{L}$ was also low at 15\% (range 9-22\%). The EAS/ ESC guidelines recommend an LDL-C target of $<2.5 \mathrm{mmol} / \mathrm{L}$ for patients at high risk, defined by a SCORE of $5-10 \%$, and $<1.8 \mathrm{mmol} /$ $\mathrm{L}$ for those at very high risk (SCORE $>10 \%$ ). Across the four studies reporting LDL-C goal achievement stratified by high/very high risk, goal achievement was greater among patients at high risk (pooled estimate, 46\%) compared with very high risk (18\%) (Fig. 2).

When comparing the 5-year period before and after the publication of the 2011 ESC/EAS guidelines, a meaningful comparison was only possible among studies reporting on individuals with established CVD. The achievement of LDLC targets over time for these studies is summarised in Supplementary Appendix 6. Compared with studies reporting LDL-C measurements taken entirely before the publication of the 2011 guidelines, achievement of both LDL-C $<1.8 \mathrm{mmol} / \mathrm{L}$ and LDL$\mathrm{C}<2.5 \mathrm{mmol} / \mathrm{L}$ was significantly higher in those with measurements made after 2011 (LDL-C $<1.8 \mathrm{mmol} / \mathrm{L}, 22 \%$ versus $15 \%$; LDL$\mathrm{C}<2.5 \mathrm{mmol} / \mathrm{L}, \quad 68 \%$ versus $61 \%$; both $p<0.001)$. reporting LDL-C goal achievement stratified by high risk and very high risk [48-51]. LDL-C low-density lipoprotein cholesterol, HR high risk, VHR very high risk

Only 12 studies reported the pre-specified secondary outcomes of MI, stroke or all-cause mortality; 10 of these included participants with established CVD, one study included participants with FH and one included participants with DM (Supplementary Appendix 7). No single study reported all of the pre-specified secondary outcomes. Among individuals with established CVD, event rates for MI (9.3-32.4 per 1000 person-years), stroke (1.2-9.5 per 1000 person-years), MACE (10.7-167.5 per 1000 person-years) and all-cause mortality (3.1-58.5 per 1000 person-years) varied widely between studies.

\section{DISCUSSION}

In this systematic review, results from 81 European observational studies suggest poor LDL-C control among patient groups at high/very high risk of cardiovascular events. Achievement of the more aggressive target of $<1.8 \mathrm{mmol} / \mathrm{L}$, as recommended in ESC/EAS 2016 guidelines [1] for individuals at very high risk (established CVD or SCORE $\geq 10 \%$ ), was especially poor, at less than $19 \%$ in both risk groups. The proportions of patients reaching the target of $<2.5 \mathrm{mmol} / \mathrm{L}$ applicable to the $\mathrm{FH}$ and SCORE 5-10\% risk groups were also suboptimal 
(15\% and $46 \%$, respectively). When comparing the 5-year period before and after the publication of the ESC/EAS guideline in 2011 (in the established CVD risk group), rates of LDL-C target achievement improved significantly over time. However, rates of LDL-C target achievement remained suboptimal in the 5-year period after the publication of the 2011 guidelines (LDL-C $<1.8,22 \%$; LDL-C $<2.5,68 \%$ ).

Several multinational surveys have examined the use of lipid-lowering therapy and LDLC goal attainment in the setting of secondary prevention [14-17]. This systematic review is the first to investigate the achievement of therapeutic targets for LDL-C in observational studies across four clinical risk categories in Europe. In high-dose statin secondary prevention trials conducted in highly selective patient populations, and despite close monitoring of adherence, which is not feasible in clinical practice, a high proportion of patients did not obtain optimal LDL-C levels. The proportion of patients achieving target levels in our study appears to be higher and highlights the discordance between trials and real-world clinical practice. Meta-analyses undertaken by the Cholesterol Treatment Trialists' (CTT) Collaboration on statin trials suggest that a $1.0 \mathrm{mmol} / \mathrm{L}$ reduction in LDL-C is associated with a relative risk (RR) of 0.90 (95\% CI 0.87-0.93) for all-cause mortality, i.e. a risk reduction of $10 \%$. Major coronary events are similarly reduced by $24 \%$ (RR $0.76,95 \%$ CI $0.73-0.79$ ) and stroke by $15 \%$ (RR 0.85, 95\% CI 0.8-0.89) [2, 3]. Importantly, these benefits were observed irrespective of baseline LDL-C, and at levels below $2 \mathrm{mmol} / \mathrm{L}$, with no evidence to suggest that low LDL-C levels result in any adverse effects to counteract the benefit. In the present study, the mean LDL$C$ value in the patient group with established CVD was $2.8 \mathrm{mmol} / \mathrm{L}$. Lowering LDL-C among this group to the $1.8 \mathrm{mmol} / \mathrm{L}$ target in the 2016 ESC/EAS guidelines would therefore result in meaningful CVD risk reductions on a population level. Our findings suggest that a significant proportion of patients at high or very high risk of CVD are being exposed to LDL-C concentrations far in excess of recommended targets. Future efforts need to address the disparity between evidence-based guidance and clinical practice and to understand the reasons behind poor LDL-C control among high-risk patients.

Suboptimal LDL-C target achievement was observed despite almost all patients with established CVD and SCORE $\geq 5 \%$ receiving statins ( $89 \%$ and $99 \%$, respectively). These findings on statin use compare favourably with a US study assessing the impact of the 2013 American College of Cardiology/American Heart Association (ACC/AHA) cholesterol guidelines, where data from the PINNACLE registry showed that $28 \%$ of eligible patients with atherosclerotic CVD did not receive any lipid-lowering medication [18]. Underutilisation of lipid-lowering therapy was also reported in an earlier cohort of patients with a history of MI in the US National Health and Nutrition Examination Survey [19]. The poor attainment of LDL-C targets observed in our study suggests that patients at very high risk are not receiving statin therapy at an adequate intensity, as suggested by single country observational studies in the UK and Spain $[20,21]$. In addition to specific targets for the risk groups we studied, the 2016 ESC/EAS guidelines advocate an absolute reduction in LDL-C of $50 \%$ in very high-risk patients with established CVD, DM with target organ damage, or SCORE $\geq 10 \%$. It is therefore possible that insufficient numbers of patients are receiving moderate-intensity or high-intensity statin treatments, defined as regimens which reduce LDL-C by $30-50 \%$ and by $50 \%$, respectively. Few data were available on the intensity of statin therapy among participants in the included studies to confirm such a clinical inertia hypothesis; however, inadequate intensity of lipid-lowering therapy in high-risk populations has been widely reported $[22,23]$. Registry data from the USA and Europe, for example, suggest that only $23-38 \%$ of patients discharged following an MI receive maximal-intensity statin therapy [24-26].

Recent randomised trials have reported additional LDL-C lowering and further reduction in CVD risk with ezetimibe, anacetrapib and two proprotein convertase subtilisin/ kexin 9 (PCSK9) monoclonal antibodies, evolocumab and alirocumab [27-32]. While evidence regarding the clinical benefit of these treatments was only available towards the end of the 
present study period, any future improvement in LDL-C control is likely to depend, in part, on greater use of non-statin therapy in patients failing to reach target LDL-C or those unable to tolerate statins. Doubling statin doses results in a further $6 \%$ reduction in LDL-C with good compliance [33]; however, side effects attributed by patients to therapy increase with dosing intensity [34]. LDL-C thresholds set out in the ESC/EAS 2011 and 2016 guidelines can therefore be used to prompt consideration of nonstatin agents to further lower LDL-C and CVD risk [35]. ESC/EAS have published guidance on the use of PCSK9 inhibitors in patients with atherosclerotic CVD and $\mathrm{FH}$, including clinical decision algorithms [36]. An observational report on primary care data in Spain suggests that around $0.3 \%$ of the adult population would be candidates for treatment with a PCSK9 inhibitor [37], according to this guidance.

Another possible explanation for low achievement of the $1.8 \mathrm{mmol} / \mathrm{L}$ LDL-C target is poor medication adherence. Even in the setting of well-designed controlled studies, discontinuation rates for lipid-lowering therapy are high. The IMProved Reduction of Outcomes: Vytorin Efficacy International Trial (IMPROVE-IT) reported that up to $40 \%$ of patients discontinued medication [29]. In large registries of patients with coronary heart disease, statin discontinuation rates are even higher, reaching $50 \%$ at 1 year [38-40]. Real-world data on the impact of good adherence (defined as proportion of days covered at least $80 \%$ ) to lipid-lowering therapy in an Italian cohort at very high $\mathrm{CV}$ risk suggests that it is associated with a three times higher probability of reaching the therapeutic LDL-C target [41]. Furthermore, clinical trial and registry data have demonstrated higher mortality rates following statin discontinuation in secondary prevention [42, 43]. Statin discontinuation, in part due to negative press $[44,45]$, has also been shown to increase the risk of myocardial infarction and cardiovascular mortality [44, 46, 47]. Irrespective of the underlying reasons for the poor achievement of ESC/EAS LDL-C targets reported here, the ESC/ EAS guidelines place considerable weight on LDL-C monitoring to measure therapeutic efficacy and patient compliance. Our findings suggest that this process is not happening effectively in clinical practice across Europe and durable strategies are needed to address gaps in lipid-lowering for primary and secondary prevention in high-risk groups. These findings are particularly relevant following the release of 2019 ESC/EAS dyslipidaemia guidelines, which recommend that LDL-C levels should be lowered as much as possible to prevent CVD [6]. Given our data suggesting that the 2016 LDL-C targets were not being achieved in patients with high/very high-risk CVD, the unmet need in this population will be even greater when assessed against the lower, more aggressive 2019 targets.

As with any systematic review, our limitations reflect those of the included studies. Information on baseline characteristics was incomplete for a large number of studies, especially those where only a subgroup of participants met our inclusion criteria. An additional limitation was our inability to determine the intensity of statin therapy or adherence to prescribed lipid-lowering therapy, both of which may influence LDL-C levels. The low percentage of patients with established CVD and SCORE $\geq 5 \%$ achieving target LDL-C levels, despite almost all patients receiving statins (89\% and $99 \%$, respectively), suggest that these patients were receiving low- or medium-intensity statins and/or were not adhering to their prescribed lipid-lowering therapy. Data on outcomes of interest were inconsistently reported across the included studies, and individual participant data were lacking, preventing meaningful analyses to examine the influence of LDL-C goal attainment on cardiovascular endpoints. Only a small number of studies involving patients with diabetes and target organ damage were identified, none of which reported achievement of the relevant LDL-C target $(<1.8 \mathrm{mmol} / \mathrm{L})$. Furthermore, only two out of seven studies in patients with diabetes and target organ damage reported achievement of the more conservative target of $<2.5 \mathrm{mmol} / \mathrm{L}$. In future studies, consistent reporting of the relevant LDL-C target achievement in different at-risk populations will allow meaningful updates to the present review and help guide efforts to improve clinical practice. 


\section{CONCLUSION}

Observational data from across Europe suggest suboptimal LDL-C control across four clinical risk categories for CVD. Those at the greatest overall risk, including those with a greater than $10 \%$ 10-year risk of fatal CVD (as assessed by SCORE) or clinically established CVD, had the lowest achievement of LDL-C targets. Attainment of more conservative targets than advocated in current 2019 EAS/ESC guidance was also poor. Our findings suggest a significant unmet need in LDL-C control among individuals at high or very high risk of CVD, and an opportunity to attenuate cardiovascular events on a population level. Given that our data suggest the 2016 LDL-C targets were not being achieved in patients with high/very high-risk CVD, the unmet need in this population will be even greater when assessed against the lower, more aggressive 2019 targets.

\section{ACKNOWLEDGEMENTS}

Funding. This study and the journal's Rapid Service and Open Access Fees were funded by Amgen (Europe) GmbH.

Medical Writing Assistance. The authors thank Jack Brownrigg and Andrew McGovern of London et al. Scientific Communications Ltd., who conducted the searches and provided medical writing assistance, and Claire Desborough of Amgen (Europe) $\mathrm{GmbH}$, who provided editorial assistance. Funding for this medical writing support was provided by Amgen (Europe) $\mathrm{GmbH}$.

Authorship. All named authors meet the International Committee of Medical Journal Editors (ICMJE) criteria for authorship for this article, take responsibility for the integrity of the work as a whole, and have given their approval for this version to be published. All authors contributed to the design of this systematic literature review and the interpretation of the data. All authors provided input to draft versions of the manuscript, had final control over data interpretation and publication content, and approved the final version.

Disclosures. Eric Bruckert has received consulting fees from AMGEN, Genfit, MSD, Sanofi, Regeneron, Unilever, Aegerion, Chiesi, Rottapharm-MEDA, Lilly, Ionis-pharmaceuticals, AKCEA. Klaus Georg Parhofer has received research grants from MSD and Sanofi; consulting fees from Aegerion, Alexion, Amgen, BerlinChemie, Boehringer-Ingelheim, MSD, Regeneron, Sanofi; speakers' fees from Amgen, BerlinChemie, MSD, Sanofi. Jose Ramon GonzalezJuanatey has nothing to disclose. Børge Nordestgaard has received consulting fees from AstraZeneca, Omthera, Sanofi, Regeneron, Ionis, Aegerion, Dezima, Amgen and Kowa. Marcello Arca has received research grants and consulting fees from Amgen, Regeneron, Sanofi, Akcea, Aegerion; research grants from Chiesi and Pfizer; consulting fees from Alfasigma. Periklis Giovas is an Amgen employee. Kausik Ray has received research grants from Pfizer, Sanofi, Regeneron, Amgen; consulting fees from Amgen, Sanofi, Regeneron, MedCo, Cerenis, Lilly, Ionis Pharma, Abbvie; speakers' fees from Kowa, AstraZeneca, Pfizer, Takeda, Boehringer Ingelheim, Algorithm, Cipla.

Compliance with Ethics Guidelines. This article is based on previously conducted studies and does not contain any studies with human participants or animals performed by any of the authors.

Data Availability. Qualified researchers may request data from Amgen clinical studies. Complete data are available at the following: https://wwwext.amgen.com/science/clinicaltrials/clinical-data-transparency-practices/.

Open Access. This article is licensed under a Creative Commons Attribution-NonCommercial 4.0 International License, which permits any non-commercial use, sharing, adaptation, distribution and reproduction in any medium or format, as long as you give appropriate credit to the original author(s) and the source, provide a link to the Creative Commons licence, and 
indicate if changes were made. The images or other third party material in this article are included in the article's Creative Commons licence, unless indicated otherwise in a credit line to the material. If material is not included in the article's Creative Commons licence and your intended use is not permitted by statutory regulation or exceeds the permitted use, you will need to obtain permission directly from the copyright holder.To view a copy of this licence, visit http://creativecommons.org/licenses/by$\mathrm{nc} / 4.0 /$.

\section{REFERENCES}

1. Catapano AL, Graham I, De Backer G, et al. 2016 ESC/EAS guidelines for the management of dyslipidaemias. Eur Heart J. 2016;37:2999-3058.

2. Cholesterol Treatment Trialists' (CTT) Collaboration, Baigent C, Blackwell L, et al. Efficacy and safety of more intensive lowering of LDL cholesterol: a meta-analysis of data from 170000 participants in 26 randomised trials. Lancet. 2010;376: 1670-81.

3. Cholesterol Treatment Trialists' (CTT) Collaborators, Mihaylova B, Emberson J, et al. The effects of lowering LDL cholesterol with statin therapy in people at low risk of vascular disease: meta-analysis of individual data from 27 randomised trials. Lancet. 2012;380:581-90.

4. Piepoli MF, Hoes AW, Agewall S, et al. 2016 European guidelines on cardiovascular disease prevention in clinical practice: the Sixth Joint Task Force of the European Society of Cardiology and Other Societies on Cardiovascular Disease Prevention in Clinical Practice (constituted by representatives of 10 societies and by invited experts) Developed with the special contribution of the European Association for Cardiovascular Prevention \& Rehabilitation (EACPR). Eur Heart J. 2016;37:2315-81.

5. Reiner Ž, Catapano AL, De Backer G, et al. ESC/EAS guidelines for the management of dyslipidaemias: the Task Force for the management of dyslipidaemias of the European Society of Cardiology (ESC) and the European Atherosclerosis Society (EAS). Eur Heart J. 2011;32:1769-818.

6. Mach F, Baigent C, Catapano AL, et al. 2019 ESC/ EAS guidelines for the management of dyslipidaemias: lipid modification to reduce cardiovascular risk: the Task Force for the management of dyslipidaemias of the European Society of
Cardiology (ESC) and European Atherosclerosis Society (EAS). Eur Heart J. 2019;41:111-88.

7. Santos RD, Waters DD, Tarasenko L, et al. A comparison of non-HDL and LDL cholesterol goal attainment in a large, multinational patient population: the Lipid Treatment Assessment Project 2. Atherosclerosis. 2012;224:150-3.

8. Conroy R, Pyörälä K, Fitzgerald A, et al. Estimation of ten-year risk of fatal cardiovascular disease in Europe: the SCORE project. Eur Heart J. 2003;24: 987-1003.

9. Stroup DF, Berlin JA, Morton SC, et al. Meta-analysis of observational studies in epidemiology: a proposal for reporting. JAMA. 2000;283:2008-12.

10. Moher D, Liberati A, Tetzlaff J, Altman DG, The PRISMA Group. Preferred reporting items for systematic reviews and meta-analyses: the PRISMA statement. PLoS Med. 2009;6:e1000097.

11. Ravnskov U. Lack of evidence for recommended low-density lipoprotein cholesterol treatment targets. Ann Intern Med. 2007;146:614 (author reply 614).

12. De Vera MA, Bhole V, Burns LC, Lacaille D. Impact of statin adherence on cardiovascular disease and mortality outcomes: a systematic review. Br J Clin Pharmacol. 2014;78:684-98.

13. Sterne JA, Hernán MA, Reeves BC, et al. ROBINS-I: a tool for assessing risk of bias in non-randomised studies of interventions. BMJ. 2016;355:i4919.

14. Mehta RH, Bhatt DL, Steg PG, et al. Modifiable risk factors control and its relationship with 1 year outcomes after coronary artery bypass surgery: insights from the REACH registry. Eur Heart J. 2008;29:3052-60.

15. Vedin O, Hagström E, Stewart R, et al. Secondary prevention and risk factor target achievement in a global, high-risk population with established coronary heart disease: baseline results from the STABILITY study. Eur J Prev Cardiol. 2013;20:678-85.

16. Yusuf S, Islam S, Chow CK, et al. Use of secondary prevention drugs for cardiovascular disease in the community in high-income, middle-income, and low-income countries (the PURE Study): a prospective epidemiological survey. Lancet. 2011;378:1231-43.

17. Kotseva K, Wood D, De Bacquer D, et al. EUROASPIRE IV: a European Society of Cardiology survey on the lifestyle, risk factor and therapeutic management of coronary patients from 24 European countries. Eur J Prev Cardiol. 2016;23:636-48. 
18. Maddox TM, Borden WB, Tang F, et al. Implications of the 2013 ACC/AHA cholesterol guidelines for adults in contemporary cardiovascular practice. J Am Coll Cardiol. 2014;64:2183-92.

19. Shah NS, Huffman MD, Ning H, Lloyd-Jones DM. Trends in myocardial infarction secondary prevention: the National Health and Nutrition Examination Surveys (NHANES), 1999-2012. J Am Heart Assoc. 2015;4:e001709.

20. Steen DL, Khan I, Ansell D, Sanchez RJ, Ray KK. Retrospective examination of lipid-lowering treatment patterns in a real-world high-risk cohort in the UK in 2014: comparison with the National Institute for Health and Care Excellence (NICE) 2014 lipid modification guidelines. BMJ Open. 2017;7:e013255.

21. de la Sierra A, Pintó X, Guijarro C, et al. Prevalence, treatment, and control of hypercholesterolemia in high cardiovascular risk patients: evidences from a systematic literature review in Spain. Adv Ther. 2015;32:944-61.

22. Toth PP, Foody JM, Tomassini JE, et al. Therapeutic practice patterns related to statin potency and ezetimibe/simvastatin combination therapies in lowering LDL-C in patients with high-risk cardiovascular disease. J Clin Lipidol. 2014;8: 107-16.

23. Simpson RJ, Tunceli K, Ramey DR, et al. Treatment pattern changes in high-risk patients newly initiated on statin monotherapy in a managed care setting. J Clin Lipidol. 2013;7:399-407.

24. Arnold SV, Spertus JA, Masoudi FA, et al. Beyond medication prescription as performance measures: optimal secondary prevention medication dosing after acute myocardial infarction. J Am Coll Cardiol. 2013;62:1791-801.

25. Arnold SV, Kosiborod M, Tang F, et al. Patterns of statin initiation, intensification, and maximization among patients hospitalized with an acute myocardial infarction. Circulation. 2014;129: 1303-9.

26. Javed U, Deedwania PC, Bhatt DL, et al. Use of intensive lipid-lowering therapy in patients hospitalized with acute coronary syndrome: an analysis of 65,396 hospitalizations from 344 hospitals participating in Get With The Guidelines (GWTG). Am Heart J. 2011;161(418-424):e3.

27. Robinson JG, Farnier M, Krempf M, et al. Efficacy and safety of alirocumab in reducing lipids and cardiovascular events. N Engl J Med. 2015;372: 1489-99.
28. Sabatine MS, Giugliano RP, Wiviott SD, et al. Efficacy and safety of evolocumab in reducing lipids and cardiovascular events. N Engl J Med. 2015;372: 1500-9.

29. Cannon CP, Blazing MA, Giugliano RP, et al. Ezetimibe added to statin therapy after acute coronary syndromes. N Engl J Med. 2015;372:2387-97.

30. Sabatine MS, Giugliano RP, Keech AC, et al. Evolocumab and clinical outcomes in patients with cardiovascular disease. N Engl J Med. 2017;2017: 1713-22.

31. HPS3/TIMI55-REVEAL Collaborative Group, Bowman L, Hopewell JC, et al. Effects of anacetrapib in patients with atherosclerotic vascular disease. N Engl J Med. 2017;377:1217-27.

32. Ridker PM, Everett BM, Thuren T, et al. Antiinflammatory therapy with canakinumab for atherosclerotic disease. N Engl J Med. 2017;377: 1119-31.

33. Law MR, Wald NJ, Rudnicka A. Quantifying effect of statins on low density lipoprotein cholesterol, ischaemic heart disease, and stroke: systematic review and meta-analysis. BMJ. 2003;326:1423.

34. Finegold JA, Manisty CH, Goldacre B, Barron AJ, Francis DP. What proportion of symptomatic side effects in patients taking statins are genuinely caused by the drug? Systematic review of randomized placebo-controlled trials to aid individual patient choice. Eur J Prev Cardiol. 2014;21:464-74.

35. Robinson JG, Ray K. Counterpoint: low-density lipoprotein cholesterol targets are not needed in lipid treatment guidelines. Arterioscler Thromb Vasc Biol. 2016;36:586-90.

36. Landmesser U, Chapman MJ, Stock JK, et al. 2017 update of ESC/EAS Task Force on practical clinical guidance for proprotein convertase subtilisin/kexin type 9 inhibition in patients with atherosclerotic cardiovascular disease or in familial hypercholesterolaemia. Eur Heart J. 2018;39:1131-43.

37. Zamora A, Masana L, Comas-Cufi M, et al. Número de pacientes candidatos a recibir inhibidores de la PCSK9 según datos de 2,5 millones de participantes de la práctica clínica real. Revista Española de Cardiología. 2018;71:1010-17.

38. Ho PM, Magid DJ, Shetterly SM, et al. Medication nonadherence is associated with a broad range of adverse outcomes in patients with coronary artery disease. Am Heart J. 2008;155:772-9.

39. Yang Y, Thumula V, Pace PF, Banahan BF, Wilkin NE, Lobb WB. Predictors of medication nonadherence among patients with diabetes in Medicare Part 
D programs: a retrospective cohort study. Clin Ther. 2009;31:2178-88.

40. Foody JM, Joyce AT, Rudolph AE, Liu LZ, Benner JS. Persistence of atorvastatin and simvastatin among patients with and without prior cardiovascular diseases: a US managed care study. Curr Med Res Opin. 2008;24:1987-2000.

41. Guglielmi V, Bellia A, Pecchioli S, et al. Effectiveness of adherence to lipid lowering therapy on LDLcholesterol in patients with very high cardiovascular risk: a real-world evidence study in primary care. Atherosclerosis. 2017;263:36-41.

42. Puri R, Libby P, Nissen SE, et al. Long-term effects of maximally intensive statin therapy on changes in coronary atheroma composition: insights from SATURN. Eur Heart J Cardiovasc Imaging. 2014;15: 380-8.

43. Navarese EP, Kowalewski M, Andreotti F, et al. Meta-analysis of time-related benefits of statin therapy in patients with acute coronary syndrome undergoing percutaneous coronary intervention. Am J Cardiol. 2014;113:1753-64.

44. Nielsen SF, Nordestgaard BG. Negative statin-related news stories decrease statin persistence and increase myocardial infarction and cardiovascular mortality: a nationwide prospective cohort study. Eur Heart J. 2015;37:908-16.

45. Matthews A, Herrett E, Gasparrini A, et al. Impact of statin related media coverage on use of statins: interrupted time series analysis with UK primary care data. BMJ. 2016;353:i3283.

46. Zhang H, Plutzky J, Shubina M, Turchin A. Continued statin prescriptions after adverse reactions and patient outcomes: a cohort study. Ann Intern Med. 2017;167:221-7.

47. Serban M-C, Colantonio LD, Manthripragada AD, et al. Statin intolerance and risk of coronary heart events and all-cause mortality following myocardial infarction. J Am Coll Cardiol. 2017;69:1386-95.

48. Eber B, Lautsch D, Fauer C, et al. Can LDL-cholesterol targets be achieved in a population at high risk? Results of the non-interventional study ACT II. Curr Med Res Opin. 2012;28:1447-54.

49. Barkas F, Milionis H, Kostapanos MS, Mikhailidis DP, Elisaf M, Liberopoulos E. How effective are the ESC/EAS and 2013 ACC/AHA guidelines in treating dyslipidemia? Lessons from a lipid clinic. Curr Med Res Opin. 2015;31:221-8.

50. Alexopoulos D, Anastasiou-Nana M, Elisaf MS, et al. A contemporary cross-sectional study on dyslipidemia management, cardiovascular risk status, and patients' quality of life in Greece: the CHALLENGE study. Int J Cardiol. 2016;217:183-9.

51. Gitt AK, Drexel H, Feely J, et al. Persistent lipid abnormalities in statin-treated patients and predictors of LDL-cholesterol goal achievement in clinical practice in Europe and Canada. Eur J Prev Cardiol. 2012;19:221-30. 